\title{
Coordinated expression of ncRNAs and HOX mRNAs in the human HOXA locus
}

\author{
Yasnory T. F. Sasaki ${ }^{a}$, Miho Sano ${ }^{a, c}$, Taishin $\mathrm{Kin}^{\mathrm{b}}$, Kiyoshi Asai ${ }^{\mathrm{b}, \mathrm{e}}$ and Tetsuro Hirose $\mathrm{a}^{\mathrm{a} \mathrm{d}_{*}}$ \\ ${ }^{a}$ Functional RNA Research Team, Biological Information Research Center, ${ }^{\mathrm{b}}$ Mathematical Modeling \\ Team, Computational Biology Research Center, National Institute of Advanced Industrial Science and \\ Technology (AIST) and 'Japan Biological Informatics Consortium (JBIC), 2-42 Aomi, Koutou, Tokyo \\ 135-0064, Japan, ${ }^{d} P R E S T O$, Japan Science and Technology Agency, Saitama 332-0012, Japan, \\ ${ }^{e}$ Graduate School of Frontier Sciences, University of Tokyo, Chiba 277-8583, Japan
}

${ }^{*}$ Corresponding author. Fax: +81-3-3599-8579.

E-mail address: tehirose@jbirc.aist.go.jp (T. Hirose)

Keywords: Noncoding RNA (ncRNA), homeotic (HOX) gene, HOXA cluster, ultra-conserved region, altemative splicing.

\begin{abstract}
In the human HOXA locus a number of ncRNAs are transcribed from the intergenic regions in the opposite direction to HOXA mRNAs. We observed that the genomic organization of genes for the ncRNAs and HOXA prote ins are highly conserved between human and mouse. We examined the expression profiles of these ncRNAs and HOXA mRNAs in various human tissues. The expression patterns of ncRNAs in human tissues coincide with those of the adjacent HOXA mRNAs that are collinearly expressed along the anteroposterior axis. This coordinated expression was observed even in transformed tumors and cancer cell lines, suggesting that the expression of ncRNAs is pre requisite for the regulated expression of HOXA genes. HIT 18844 ncRNA transcribed from the most upstream position of the HOXA cluster possesses an ultra-conserved short stretch which potentially forms an evolutionarily conserved secondary structure. Our data suggest a critical role for ncRNAs in the regulation of HOXA genes expression.
\end{abstract}


Recent high-throughput transcriptome analyses have revealed that the mammalian genome produces much greater numbers of transcripts than previously estimated [1,2]. A class of newly identified transcripts unlikely to encode functional polypeptides despite the presence of both 5 ' cap and 3' poly (A) tail, have been termed noncoding RNAs (ncRNAs). NcRNAs are widespread across the genome; however, their function largely remains enigmatic with a few exceptions. Some of ncRNAs are known to participate in gene silencing mechanisms such as imprinting [3] or dosage compensation [4] and other events [5]. Recently, a number of examples have arisen to suggest that the cotranscription of noncoding transcripts influences neighboring gene transcription. The mechanism, so-called transcriptional interference, has been primarily reported in yeast SER3 gene where an upstream transcript interferes with SER3 transcription [6]. Similar inhibitory effects may be applied to the regulation of yeast IME4 gene by the antisense ncRNA required for initiating meiosis [7]. In Drosophila, nonoverlapped ncRNAs repress the transcription of the Ultrabithorax gene, presumably by transcription interference mechanism [8]. The common assertion regarding transcription inereference is that the interfering transcription process, but not the transcript itself, is important to affect the transcription of the neighboring coding genes. On the other hand, the transcription products of intergenic noncoding regions can activate a certain gene locus by counteracting silencing with Polycomb group proteins [9].

In a few cases, ncRNA itself plays a role with its own sequence to interact with transcription factors or chromatin remodeling factors to regulate adjacent gene transcription. The human dihydrofolate reductase (DHFR) gene is repressed by a noncoding interfering transcript from the upstream altemative promoter, in which the ncRNA may induce the dissociation of the preinitiation complex from the DHFR major promoter by directly interacting with general transcription factor IIB [10]. Sanchez-Elsner et al. reported that noncoding transcripts of cis-regulatory elements recruit chromatin-associated protein Ash1 to a target Ultrabithorax gene locus, an observation that challenges the regulatory mechanism through transcription interfe rence described above [11].

HOX genes encode a family of transcription factors that play a central role in establishment and maintenance of cellular identity in embryogenesis. In mammals, $39 \mathrm{HOX}$ genes are divided into four separate clusters, A, B, C, and D, in different chromosomes. Expression of HOX genes is highly coord inated both in space and time. Arrangement of HOX genes in the chromosome is in the same order as their expression along the anteroposterior axis and in the same order as their temporal expression during development (Colinearity rule) [12, 13]. Colinear regulation of HOX genes is essential not only for developing cells to acquire identity [12] but also for cells in adult tissue to maintain identity $[14,15]$. In addition to the ir roles in development, it is known that HOX genes are often dys regulated in leukemias [14-16]. Thus, the HOX genes have dual roles in development and in carcinogenesis.

We recently characterized the expression profiles of 158 ncRNAs originally selected from a large 
dataset of the human transcriptome (H-InvDB, http://www.h-invitational.jp). We found an ncRNA (HIT18844) that is predominantly expressed in prostate and placenta, resident $331 \mathrm{bp}$ upstream of the HOXA13 gene, and oriented in a head-to-head direction. The location of the HIT18844 ncRNA gene is situated as the most 5 '-end transcription unit of the HOXA13 gene cluster. Here, we identified additional ncRNAs in the intergenic regions of human and mouse HOXA gene clusters. They are aligned at equivalent positions relative to neighboring HOX genes and are transcribed from the opposite strand of the HOX genes. The ncRNA expression profiles in human tissues and tumor cells appear to be very similar to those of adjacent HOX genes.

\section{Materials and Methods}

Identification of ncRNAs in human and mouse HOXA clusters.

The human and mouse HOXA cluster, as well as the other HOX clusters, were manually inspected using UCSC genome browser [17] to identify ncRNAs in the intergenic regions. Sequence conservation was searched with the BLAST program. Searches for conserved genomic regions and prediction of secondary RNA structure were performed with RNAz program which is custom-built in the UCSC genome browser on our website (http://www.ncRNA.org/, [18, 19]).

Reverse transcription-quantitative polymerase chain reaction (RT-qPCR)

Total RNAs from adult human tissues, tumors, and cancer cell lines were purchased from Clontech and Ambion. Total RNA $(1 \mu \mathrm{g})$ was reverse transcribed using the QuantiTect reverse transcription kit (Qiagen). Aliquots of CDNA were subjected to real time PCR, performed using a LightCycler 480 SYBR Green I Master (Roche Diagnostics) following the manufacturer's protocol. Information for primers used in this study is available upon request. To estimate relative abundance among ncRNAs, we used the "cross point" (CP) value as a reference. CP is the point at which the fluorescence of a sample rises above the background fluorescence and is given in number of amplification cycles. The CP of a sample depends on the initial concentration of target DNA in the sample.

\section{Results and Discussion}

Orthologous relationships between human and mouse ncRNAs in the HOXA cluster.

We recently identified a human cDNA clone HIT18844 (AK093987) as a putative ncRNA specifically expressed in prostate and placenta (Sasakiet al, submitted). This ncRNA is mapped at the 5'end of the HOXA cluster, near the HOXA13 gene, and is transcribed from the DNA strand opposite the HOXA genes. In the vicinity of HIT18844, another ncRNA (HIT91045; $\underline{\text { BC025338) }}$ ) resides in a 
region partially overlapped with HOXA11, in the antisense orientation [20]. Further manual inspection of 3 ' regions of the HOXA locus yielded three additional groups of ncRNAs transcribed in the same orientation as the other ncRNAs. As summarized in Figure 1A, each ncRNA is located in intergenic regions between HOXA1 and A2 (HIT06113, AK022839; HIT92376, BC031342), between HOXA3 and A4 (HIT12844, AK056230; HIT94153, BC035889) and between HOXA6 and A7 (HIT16800, AK091933), respectively. Simultaneous survey of the mouse HOXA locus revealed the presence of putative ncRNAs in the corresponding locus (Fig. 1A). Two schematics in Figure 1A depict a remarkable conservation between human and mouse genomes in many aspects: the ncRNAs 1) are transcribed from the opposite strand in terms of HOX mRNAs, 2) reside in the intergenic region except for that distal to HOXA13, and 3) have putative counterparts. For example, human ncRNA HIT06113

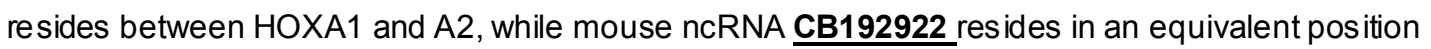
(Fig. 1A). These ncRNAs are trans cribed in the same orientation, opposite to HOXA mRNAs. This orthologous relationship between human and mouse implies functional constraint; these ncRNAs are likely to play a role in the regulation of HOX gene expression.

\section{Coordinated expression of ncRNAs and neighboring HOX mRNAs in human HOXA cluster.}

The expression of HOX mRNAs is spatio-temporally regulated in a very strict manner [12, 13]. We investigated the tissue distribution of ncRNAs in HOXA locus by RT-qPCR to determine whether the expression pattem of the ncRNAs reflects those of the neighboring HOX mRNAs. cDNAs from HOXA mRNAs and ncRNAs were amplified from at least one of 10 different human tissue, a result which experimentally demonstrated the existence of those ncRNAs. As shown in Figure 1B, the tissue-distribution pattern and the relative abundance of ncRNAs indicated that a distinct anterior-posterior gradient of expression was operative in ncRNAs, as well as in HOX mRNAs. Brain, heart and liver tissue appeared to be less active with respect to HOXA gene expression. In the other tissues, each ncRNA exhibited a characteristic expression pattern that resembled flanking HOX mRNAs patterns (Fig. 1B, compare the expression pattern between HOXA1 and HIT06113, HIT94153 and HOXA4, HOXA11 and HIT91045, and HOXA13 and HIT18844). In thymus, a thoracic tissue, there was a stream of expression from HOXA1 to HOXA4. In lung, another tho racic tissue, the stream was shifted slightly distal, following the relative posterior shift in the location of the tissue. It is important to note that ncRNAs were also co-regulated with HOXA genes in these tissues. This phenomenon is congruous with the "colinearity rule". In kidney, abdominal tissues, the expression of the ncRNAs and HOX mRNAs was rather broad, and a higher level of expression was scored for those transcripts nested in the middle of HOXA locus. In placenta and prostate, lower abdominal tissues, the HOX genes with greater numbers and nearby ncRNAs were highly expressed. This expression pattern of greater numbers of HOX genes in placenta and prostate was confirmed by Northern blot analysis (Supplemental Fig. 1). The data clearly demonstrate that the expression of 
ncRNA accompanies that of HOX mRNAs in the vicinity. In other words, the expression of ncRNAs is collinearly regulated.

Judging from the cross-point (CP) value of RT-qPCR, the abundance of ncRNAs appeared to be lower than that of flanking HOX mRNAs (data not shown). We conducted absolute quantification for some of the target RNAs ( e.g. ratio of HOXA13 to HIT18844: 6.2 in HeLa cell, 3.6 in colon. The result of northem blot hybridization also supports the above notion (Supplemental Fig. 1). It is possible that the ncRNAs are less stable than HOX mRNAs. It is consistent with the evidence that a number of ncRNAs play a role during transcription, thereby the posttranscriptionally accumulated RNA may not be functional. Alternatively, the res pective promoter streng th may reflect the resultant accumulation of HOX mRNAs and ncRNAs levels, which may be important for the proper control of the HOX gene expression.

The coordinated expression pattern is maintained in tumor tissues as well as cell lines.

To further confirm the coord inated expression of ncRNAs and HOX mRNAs in the HOXA locus, we expanded RT-qPCR analysis to normal and tumor human tissues and various human cell lines. We concentrated our attention on two late HOXA genes, HOXA11 and HOXA13, and the ir ncRNA partners, HIT91045 and HIT18844, respectively. We first confirmed the previously reported results [21] in which the late HOXA cluster (HOXA7 to HOXA13) was aberrantly activated in cervical carcinoma HeLa cells (Fig. 2A). We observed that both ncRNA partners were also highly expressed in HeLa cells. The late HOXA genes and their ncRNA partners were expressed at low levels (less than $7 \%$ of the levels in HeLa cell) in normal and tumor tissues derived from lung, kidney and ovary (Fig. 2A). In contrast, they were highly expressed ( $>7$-fold relative to the level in lung) in uterus and colon, which are lower abdominal tissues. Most importantly, a clear coincidence was observed in the expression of the paired HOX mRNAs and their ncRNAs in both normal and tumor tissues.

We then carried out expression profile analysis of the entire HOXA cluster. Figure $2 \mathrm{~B}$ and Supplemental Figure 2 clearly show that tissue-specificity of ncRNAs resembled that of HOX mRNAs in various cell lines. Most striking, the expression pattems in T24 and PC3 cells can be superimposed reciprocally (Fig. 2B and Supplemental Fig. 2). T24 and PC3 cells are bladder carcinoma and prostate adenocarcinoma cell lines, respectively. Both cell lines originated from tissues in a position near the end of the urogenital system, where HOXA11 and HOXA13 and their ncRNA partners are predominantly expressed.

Recent findings have shown that Hox genes are abnomally expressed in a variety of tumors [14-16]. Although the relationship between this abnormality and the pathology of these cancers remains elusive, Hox genes can determine the identity of malignant ovarian epithelium cells [22]. There is an apparent tendency that expression levels of both ncRNA and mRNA decline in tumor tissues as compared to nomal tissues. We found that expression profiles are not uniform between 
patients of kidney tumors and patients of uterine tumors. Further experiments aimed to explore this discrepancy and clarify the above argument, should be designed to increase the number of specimens and compare nomal and malignant specimens obtained from same individuals. HOX proteins are involved in cell renewal and in the normal physiological changes that occur during adult life. For example, expression of Hox9 paralogues expression in mammary gland changes in response to pregnancy [23]; and Hoxa10 expression in the uterus varies with the reproductive cycle [24]. Therefore, it is of great interest to determine whether the dynamic changes of ncRNA expression coincide with alterations of HOX expression during lactation and ovulation.

An ultra-conserved region is transcribed as a 5'end ncRNA in human and mouse HOXA clusters.

We found that a stretch within HIT18844 ncRNA exhibited sequence conservation in the syntenic regions among various mammalian species. The entire stretch is $265 \mathrm{bp}$ in length, and maps to a position $1.8 \mathrm{~kb}$ upstream of the HOXA13 gene, and 1568 bases from the 5' terminus of HIT18844 ncRNA in human (Fig. 3A). It is highly conserved among mammals including human, chimpanzee, Rhesus monkey, dog, rabbit, rat, mouse and opossum with identities of $65 \sim 99 \%$. Comparative computational approach using RNAz program [18] revealed that this prominently conserved region scored the highest SVM RNA-class probability $(P=1.000)$ and potentially form a characteristic secondary structure (Fig. 3B). In addition, other vertebrates such as chicken, zebrafish and fugu exhibit sequence similarity with $>87 \%$ identity over a core 47 bp sequence where characteristic substitutions occur to conserve this secondary structure (Supplemental Fig. 3). These evidences strongly support that HIT 18844 ncRNA possesses a structural RNA motif that may be functionally significant.

EST database searches to identify more transcripts containing the correspond ing sequence yielded four additional entries from mouse transcrip tome (AK033508, BB624924, BC046496, and BF57982), all of which was derived from colon. Interestingly, the first two clones contain the ultra-conserved region, whereas the remaining two clones lack the region (Fig. $3 \mathrm{C}$ ). The presence of stereotypic splice accepter/donor sites in the clones having the ultra-conserved region provides evidential support of altemative splicing (Fig. 3C). These sites are well conserved among all mammalian sequences examined, except for rabbit. In particular, the donor site immediately upstream of the ultra-conserved region is conserved not only in all mammals, including rabbit and opossum, but also in chicken. These observations strongly suggest that the transcripts are sorted by alternative splicing with some biological meaning. Altematively, because the ultra-conserved region has potential to form an evolutionarily conserved secondary structure and lies in the tip of the stem structure, we posit that a common RNA binding protein(s) recognizes the RNA structure for the function of HIT18844 ncRNA.

It has become widely accepted that noncoding regions of the genome, such as intergenic regions, are transcribed and show certain levels of conservation [25]. The ultra-conserved region within 
HIT18844 ncRNA may have a biological function as a structural ncRNA, since the region containing the sequence is transcribed, may form secondary structure and is subject to altemative splicing. Alte matively, it may act as a cis-regulatory DNA element at the 5'end of HOXA cluster, like enhancer elements located around various transcription factor genes [26]. During the course of our research, Sessa et al. reported 'opposite strand transcription' from the intergenic regions in the human HOXA cluster [27]. They propose a model in which noncoding transcription is accompanied by changes in the histone code, which in tum causes chromatin remodeling and maintenance of an active status of the HOXA cluster.

In summary, we found a battery of ncRNAs aligned in intergenic regions of the HOXA cluster. The expression of those ncRNAs is coordinately regulated with HOX mRNAs in their respective vicinity. This coordinate expression pattem is consistent in tumor tissues as well as in cancer cell lines. Our results are largely in accordance with a recently proposed concept in which noncoding RNA transcription maintains the corresponding chromatin in a transcriptionally active state by preventing binding of repressive prote in complexes from the cis-regulatory element $[9,27]$. In addition to this hypothesis, we opine that the ncRNA located upstream of the HOXA13 gene may have an additional and/or alternative function regarding the sequence conservation and the existence of splicing variants. Further analysis of the role of ncRNAs in the regulation of collinear HOX gene expression will provide insights into the mechanism by which ncRNAs participate in gene activation and silencing.

\section{Acknowledgments}

The authors thank Drs. K. Watanabe and T. Ideue for valuable discussion and encouragement. M. Nagai and M. Togawa are acknowledged for their assistance. This research was supported by the New Energy and Industrial Technology Development Organization (NEDO), PRESTO program grant from the Japan Science and Technology agency and the Ministry of Education, Culture, Sports, Science, and Technology of Japan.

\section{References}

[1]A.T. Willingham and T.R. Gingeras, TUF love for “junk” DNA, Cell 125 (2006) 1215-1220.

[2] P. Carninci, Tagging mammalian transcription complexity, Trends Genet, 22 (2006) 501-510.

[3] F. M. Pauler and D.P. Barlow, Imprinting mechanisms -it only takes two, Genes Dev. 20 (2006) 1203-1206. 
[4] E. Heard and C.M. Disteche, Dosage compensation in mammals: Fine-tuning the expression of the X chromosome, Genes Dev. 20 (2006) 1848-1867.

[5] M. Zaratuegui, D. V. Irvine and R.A. Martienssen, Noncoding RNAs and gene silencing, Cell 128 (2007) 763-776.

[6] J.A. Martens, L. Laprade and F. Winston, Intergenic transcription is required to repress the Saccharomyces cerevisiae SER3 gene, Nature 429 (2004) 571-574.

[7] C.F. Hongay, P.L Grisafi, T. Galitski and G.R. Fink, Antisense transcription controls cell fate in Saccharomyces cerevisiae, Cell 127 (2006) 735-745.

[8] S. Petruk, Y. Sedkov, K.M. Riley, J.Hodgson, F. Schweisguth, S. Hirose, J.B. Jaynes, H.W. Brock and A. Mazo, Transcription of bxd noncoding RNAs promoted by Trithorax represses Ubx in cis by transcriptional interference, Cell 127 (2006) 1209-1221.

[9] S. Schmitt, M. Prestel and R. Paro, Intergenic transcription through a Polycomb group response element counteracts silencing, Genes Dev. 19 (2005)697-708.

[10] I. Martianov, A. Ramadass, A. Serra Barros, N. Chow, and A. Akoulitchev, Repression of the human dihydrofolate reductase gene by a non-coding interfering transcript. Nature 445 (2007): $666-670$.

[11] T. Sanchez-Elsner, D. Gou, E. Kremmer and F. Sauer, Non-coding RNAs of trithorax response elements recruit Drosophila Ash1 to Ultrabithorax, Science 311 (2006) 1118-1123.

[12] M. Kmita and D. Duboule, Organizaing axes in time and space; 25 years of colinear tinkering, Science 301 (2003) 331-333.

[13] J.C. Pearson, D. Lemons and W. McGinnis, Modulating HOX gene functions during animal body patterning, Nat. Rev. Genet. 6 (2005) 893-904.

[14] R. Morgan, HOX genes: a continuation of embryonic patterning? Trends Genet. 22 (2006) 67-69.

[15] Y. Takahashi, J. Hamada, K. Murakawa, M. Takada, M. Tada, I. Nogami, N. Hayashi, S. Nakamori, M. Monden, M. Miyamoto, H. Katoh and T. Moriuchi, Expression profiles of $39 \mathrm{HOX}$ genes in normal 
human adult organs and anaplastic thyroid cancer cell lines by quantitative real-time RT-PCR system, Exp. Cell Res. 293 (2004) 144-153.

[16] D. Bansal, C. Scholl, S. Frohling, E. McDowell, B.H. Lee, K. Dohner, P. Ernst, A.J. Davidson, G.Q. Daley, L.I Zon, D.G. Gilliland and B.J.P. Huntly, Cdx4 dysregulate HOX gene expression and generates acute myeloid leukemia alone and in cooperation with Meis $1 \mathrm{a}$ in a murine model, Proc. Natl. Acad. Sci. USA, 103 (2006) 16924-16929.

[17] R.M. Kuhn, D. Karolchik, A.S. Zweig, H. Trumbower, D.J. Thomas, A. Thakkapallayil, C.W. Sugnet, M. Stanke, K.E. Smith, A. Spiel, K.R. Rosenbloom, B. Rhead, B.J. Raney, A. Pohl, J.S. Pedersen, F. Hsu, A.S. Hinrichs, R.A. Harte, M. Diekhans, H. Clawson, G. Bejerano, G.P. Barber, R. Baertsch, D. Haussler and W.J. Kent, The UCSC genome browser database: update 2007, Nucleic Acids Res. 35 (2007) D668-673.

[18] S. Washietl, I.L. Hofacker, M. Lukasser, A. Huttenhofer and P.F. Stadler, Mapping of conserved RNA secondary structures predicts thousands of functional noncoding RNAs in the human genome, Nat. Biotech. 23 (2005) 1383-1390.

[19] T. Kin, K. Yamada, G. Terai, H. Okida, Y. Yoshinari, Y. Ono, A. Kojima, Y. Kimura, T. Komori and K. Asai, fRNAdb: a platform for mining/annotating functional RNA candidates from non-coding RNA sequences, Nucleic Acids Res. 35 (2007) D145-148.

[20] S.S. Potter and W.W. Branford, Evolutionary conservation and tissue-specific processing of Hoxa11 antisense transcripts, Mammal. Genome 9 (1998) 799-806.

[21] M.G. Guenther, R.G. Jenner, B. Chevalier, T. Nakamura, C.M. Croce, E. Canaani and R.A. Young, Global and Hox-specific roles for the MLL1 methyltransferase, Proc. Natl. Acad. Sci. USA 102 (2005) 8603-8608.

[22] W. Cheng, J. Liu, H. Yoshida, D. Rosen and H. Naora, Lineage infidelity of epithelial ovarian cancers is controlled by HOX genes that specify regional identity in the reproductive tract, Nat. Med. 11 (2005) 531-537.

[23] F. Chen and M.R. Cappeci, Paralogous mouse Hox genes, Hoxa9, Hoxb9, and Hoxd9, function together to control development of the mammary gland in response to pregnancy, Proc. Natl. Acad. Sci. USA 96 (1999) 541-546. 
[24] I. Satokata, G. Benson and R. Maas, Sexually dimorphic sterility phenotypes in Hoxa10-deficient mice, Nature 374 (1995) 460-463.

[25] P. Khaitovich,J. Kelso, H. Franz, J. Visagie, T. Giger, S. Joerchel, E. Petzold, R.E. Green, M. Lachmann and S. Pääbo, Functionality of Intergenic transcription: An evolutionary comparison, PLoS Genet. 2 (2006) 1590-1598.

[26] G.K. McEwen, A. Woolfe, D. Goode, T. Vavouri, H. Callaway and G. Elgar, Ancient duplicated conserved noncoding elements in vertebrates: A genomic and functional analysis, Genome Res. 16 (2006) $451-465$.

[27] L. Sessa, A. Breiling, G. Lavorgna, L. Silvestri, G. Casari and V. Orlando, Noncoding RNA synthesis and loss of Polycomb group repression accompanies the collinear activation of the human HOXA cluster, RNA 13 (2007) 1-17.

\section{Figure legends}

Figure 1.

(A) Orthologous relationships between human and mouse ncRNAs in the HOXA cluster. Genomic arrangement of the HOXA genes and ncRNAs is shown. Orientation of transcription is indicated by arrowheads. Length of RNAs is in scale except for those illustrated by arrowheads, which are shorter than they appear in the scheme. (B) Expression profile of ncRNAs and HOXA mRNAs in the HOXA cluster. Relative abundance of each target RNA was determined by RT-qPCR from 10 different human tissues. For each experimental set, the resultant values were normalized and sorted in order of relative position of tissues along the body axis (to $p$ to bottom). The experimental datasets were readjusted among different target RNAs according to the CP and is presented as a percentage of the maximum value. Resultant data are arranged according to their locus in the cluster (left to right: proximal to distal) so that the variances in relative abundance are comparable. Data were processed from three independent experiments.

Figure 2

(A) Coordinated expression of HOXA11 mRNA- and HOXA13 mRNA-ncRNA pairs in normal and tumor tissues. Relative abundance of each target RNA was determined by RT-qPCR, and collected data were processed as in Fig. $1 \mathrm{~B}$ with the exception that data output was changed to 3-D histogram making pair-wise comparison easier: HOXA11-HIT91045 pair and HOXA13-HIT18844 pair. (B) 
Coordinated expression pattern of HOXA11 mRNA- and HOXA13 mRNA-ncRNA pairs in cancer cell lines. Data were collected from 9 different human cell lines: HeLa; cervical adenocarcinoma, HEK293; embryonic kidney, Jurkat; T-cell leukemia, HL60; promyelocytic leukemia, K562; leukemia, MCF-7; breast adenocarcinoma, HEPG2; liver carcinoma, T24; bladder carcinoma, PC3; prostate adenocarcinoma. Data were processed from two independent experiments.

Figure 3

The conserved region within HIT18844 ncRNA (A) A genomic sequence alignment of the conserved region within human HIT18844 ncRNA among 8 mammals. Ultra-conserved region is shaded. Possible base pairings are annotated on the bottom line (shown as pair symbol). (B) A graphical representation of RNA secondary structure of the conserved region. Two arrowheads point start and end position of the ultra-conserved region. (C) The presence of the putative splicing variant that lacks the ultra-conserved region. A schematic illustrates an alternative splicing pattem in the following three cDNAs focusing upon the ultra-conserved region (shown as a black box). AK093987 (human) and

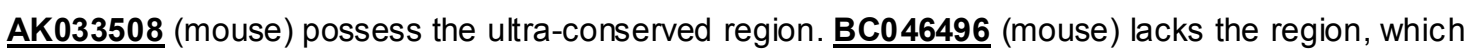
may be removed by splicing. The border sequences (GT-AG) of the removed region are shown. It should be noted that HIT18844 is likely to be an internally primed, truncated cDNA product, since a 24-consecutive polyA stretch is found in the genome immediately downstream of its 3 ' terminus. 
Figure legends for Supplemental Figures.

Supplemental Figure 1

Expression profiling of the late HOXA cluster gene. Tissue-specificities of HOXA11 and A13 mRNAs and HIT91045 and HIT18844 ncRNAs were examined by northem blot hybridization. Ten $\mu \mathrm{g}$ of total RNA from 11 human tissues and HeLa cell was applied to each lane of a $0.8 \%$ agarose gel containing $2 \%$ formaldehyde. cDNA fragments corresponding to the above HOXA

mRNAs and ncRNAs were ${ }^{32} \mathrm{P}$-labeled and probed. Filled arrowheads point at the position of the expected size. Open arrowhead in the HIT18844 panel indicates the position of detected bands different from the expected size.

\section{Supplemental Figure 2}

Relative abundance of ncRNAs and HOX mRNAs in various human cancer cell lines. Sample identity in each panel, from left to right: HeLa, HEK293, Jurkat, HL60, K562, MCF7, HepG2, T24 and PC3. The expression profile of certain RNA exhibits a similar pattern to those of other RNAs nearby in the HOXA locus. Each profile appears to have a "signature" with which it can be categorized into one of $6 \mathrm{groups}$. This may indicate the presence of subdomains in the chromatin structure of the HOXA locus.

\section{Supplemental Figure 3}

The ultra-conserved region lies in the HIT18844 ncRNA. A genomic sequence alignment of the conserved region among 11 vertebrates: human, chimpanzee, Rhesus monkey, dog, rabbit, rat, mouse, opossum, chicken, zebrafish and fugu. Possible base pairings are annotated on the bottom line. The percent identity of these vertebrate genomic sequences against human sequence are 100\%, chimpanzee; $97.9 \%$, Rhesus monkey; 93.6\%, dog; $97.9 \%$, rabbit; $93.6 \%$, rat; $93.5 \%$, mouse; $93.6 \%$, opossum; $87.2 \%$, chicken; $86.7 \%$, zebrafish and $93.5 \%$ fugu, respectively. Consistent mutation or base substitution outside of base parings are shaded whereas base substitution that dis rupts base parings is boxed. 


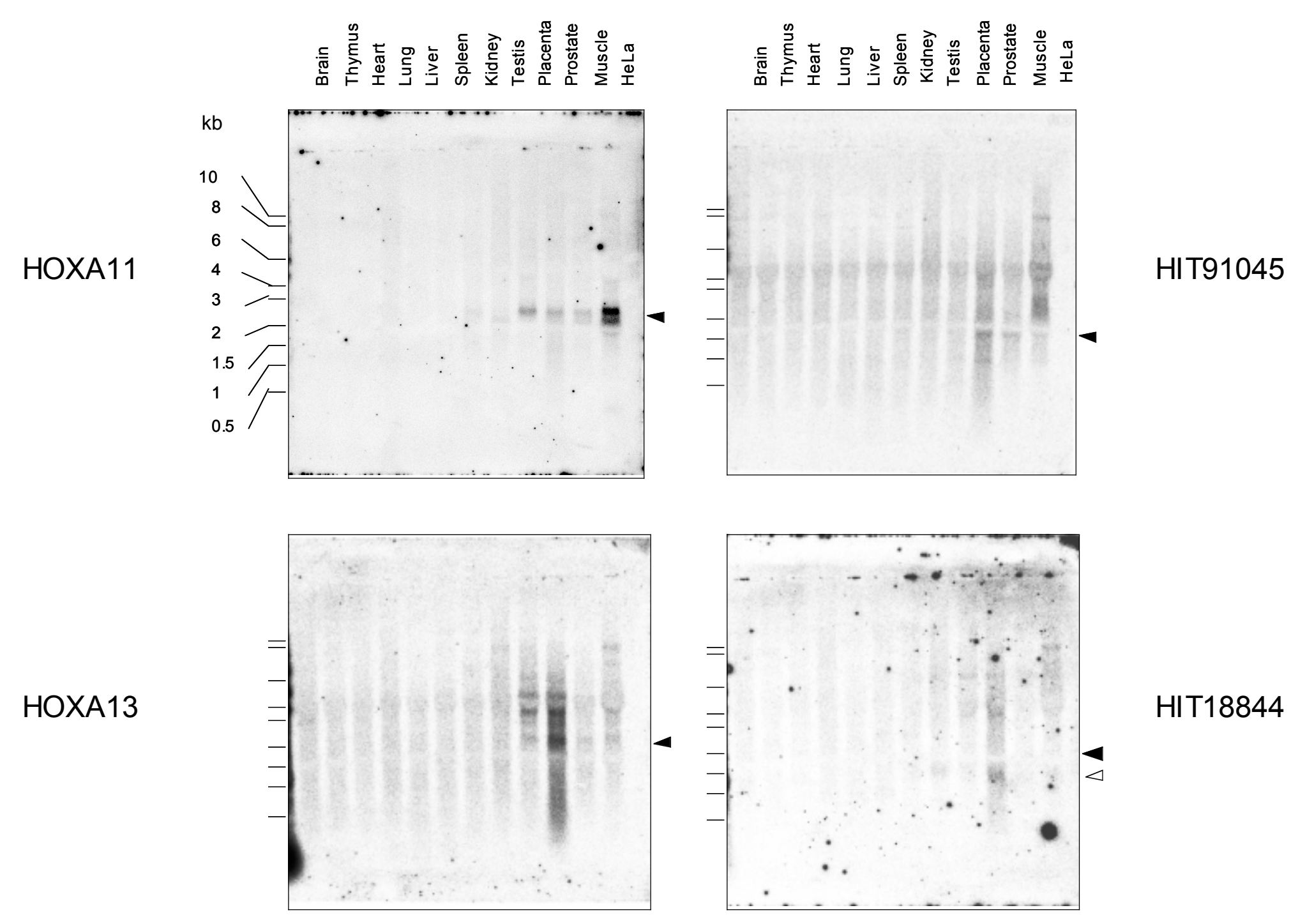

Sasaki et al. Supplemental Fig. 1 
A1

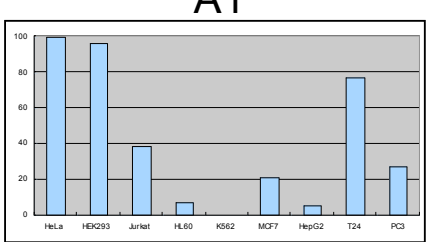

06113

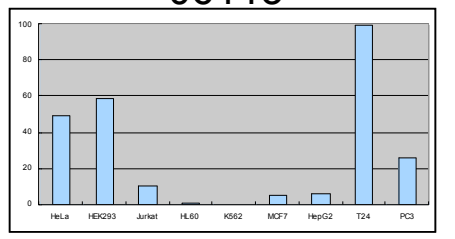

92376

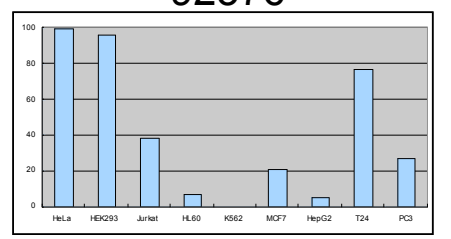

A2

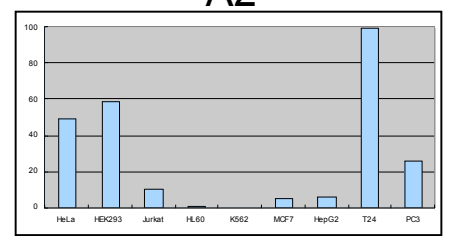

A3

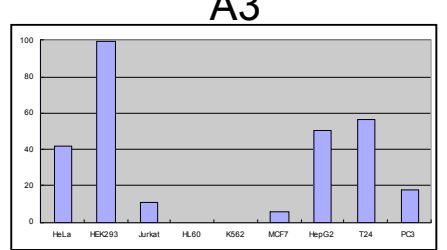

12844

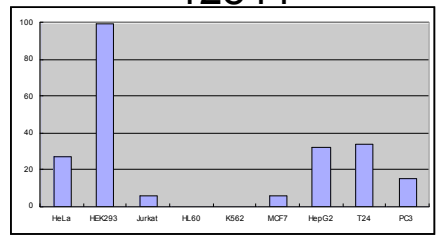

94153

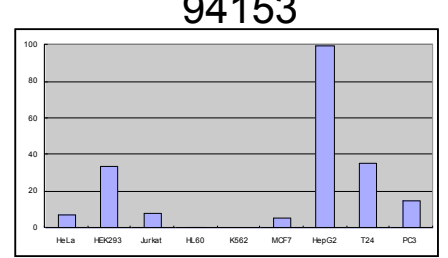

A4

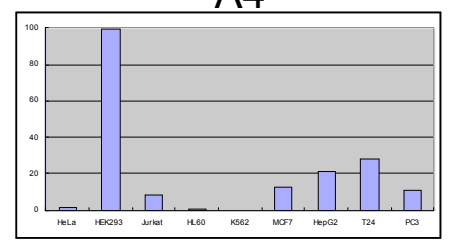

A5

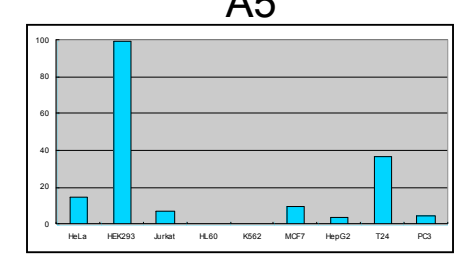

A6

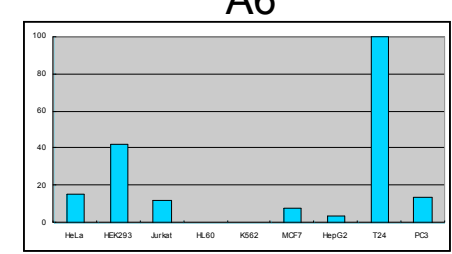

16800

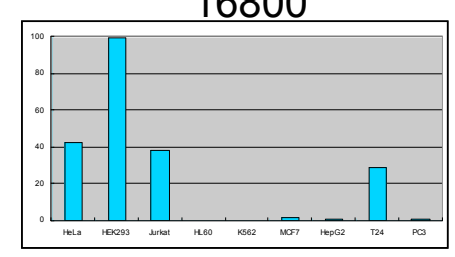

A7

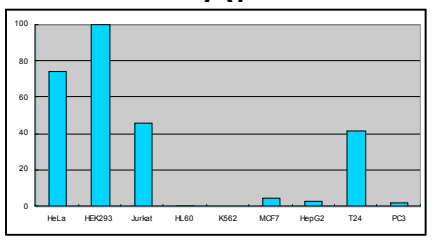

A9

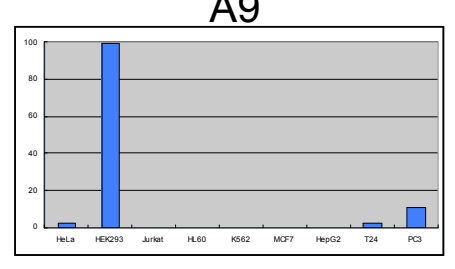

A10

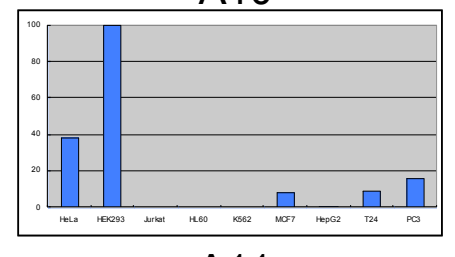

A11

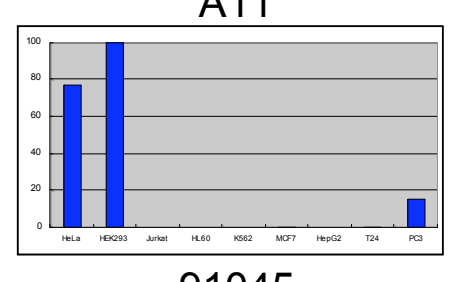

91045

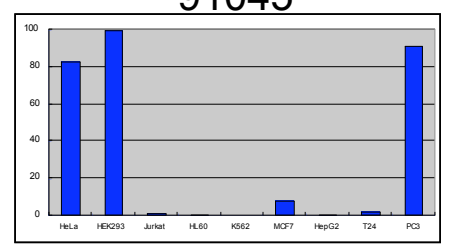

A13
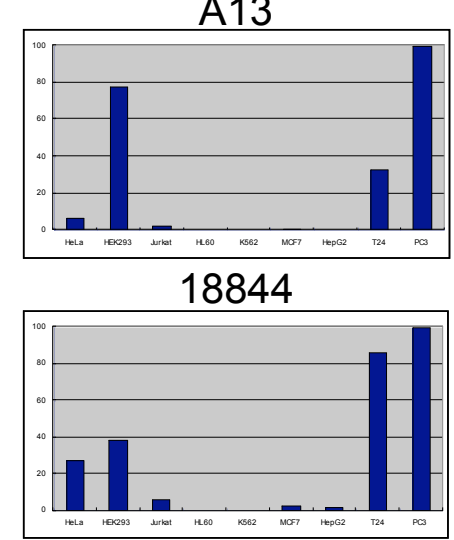

rRNA

nililull

Sasaki et al. Supplemental Fig. 2 


\section{chr7:27014964-27015010}

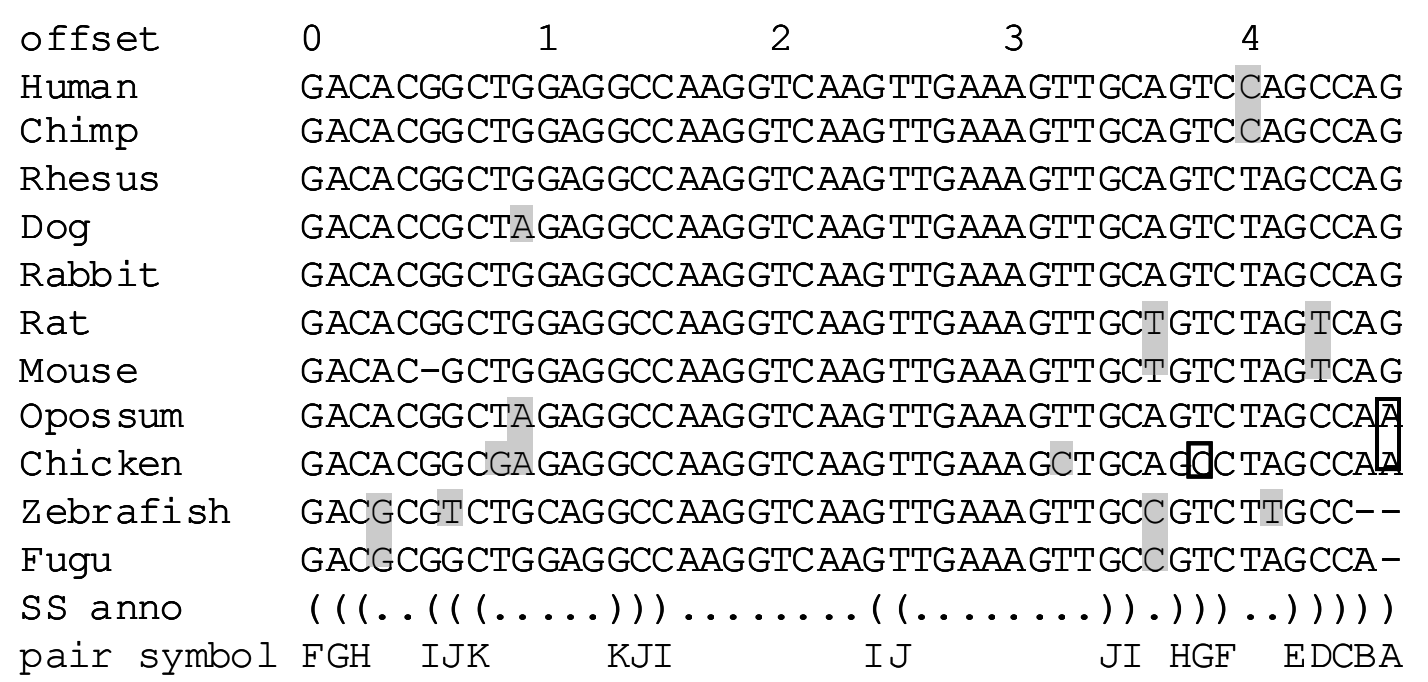


A

human chr7: 26900K - 27010K

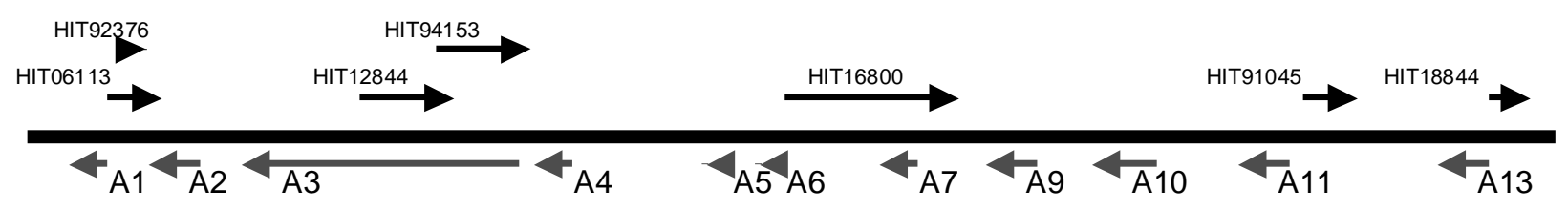

mouse chr6: 52100K - 52210K

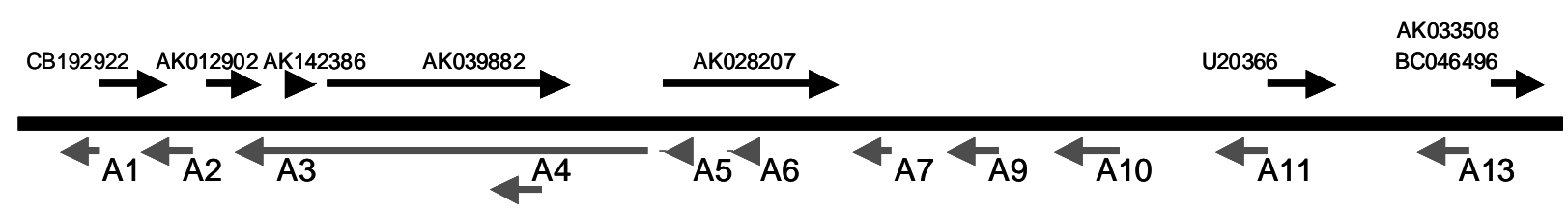

B

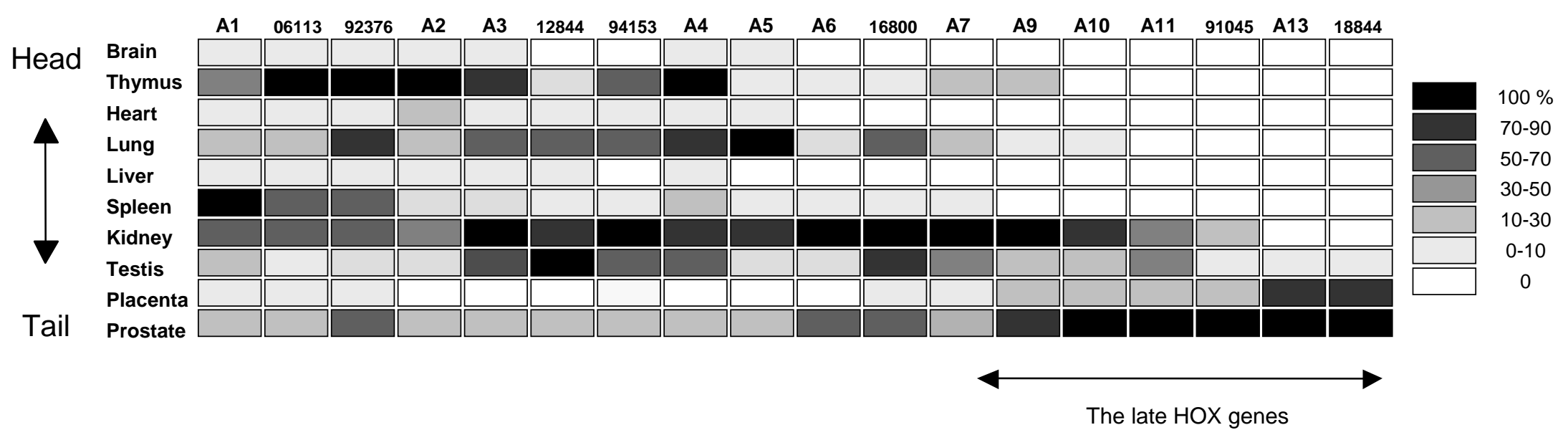

Sasaki et al. Fig. 1 


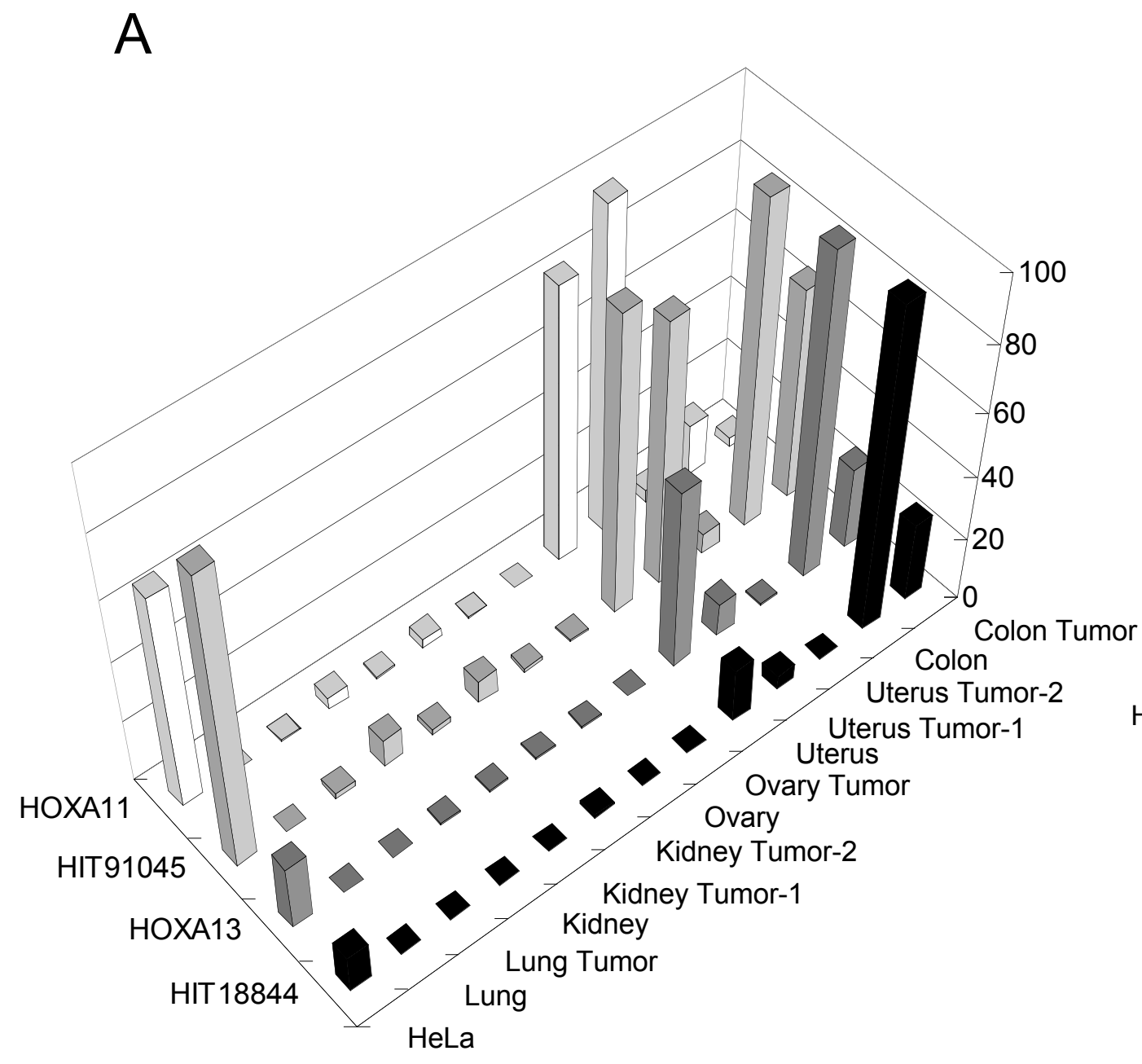

B

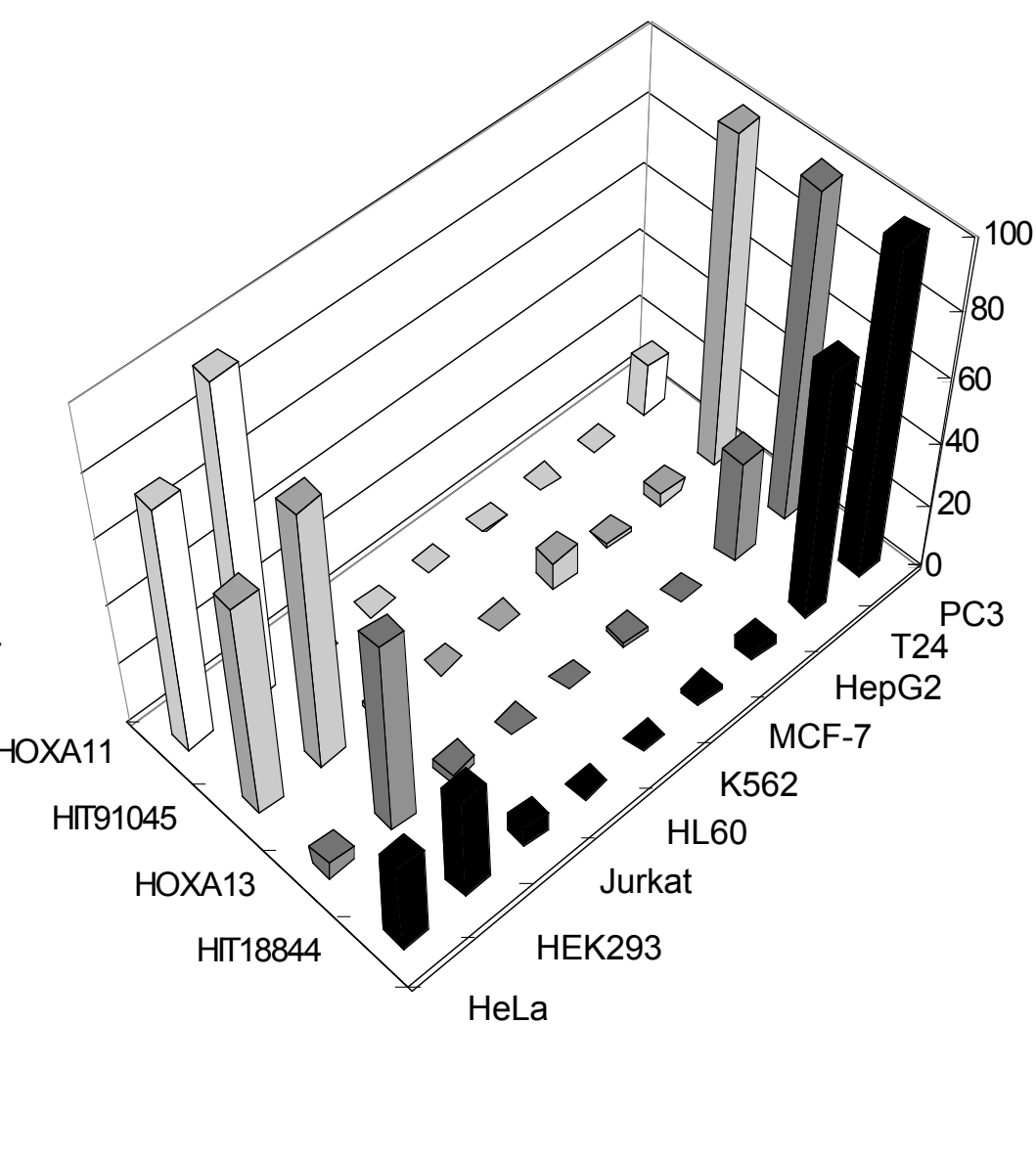

Sasaki et al. Fig. 2 
A

chr7:270 $14808-27015072$

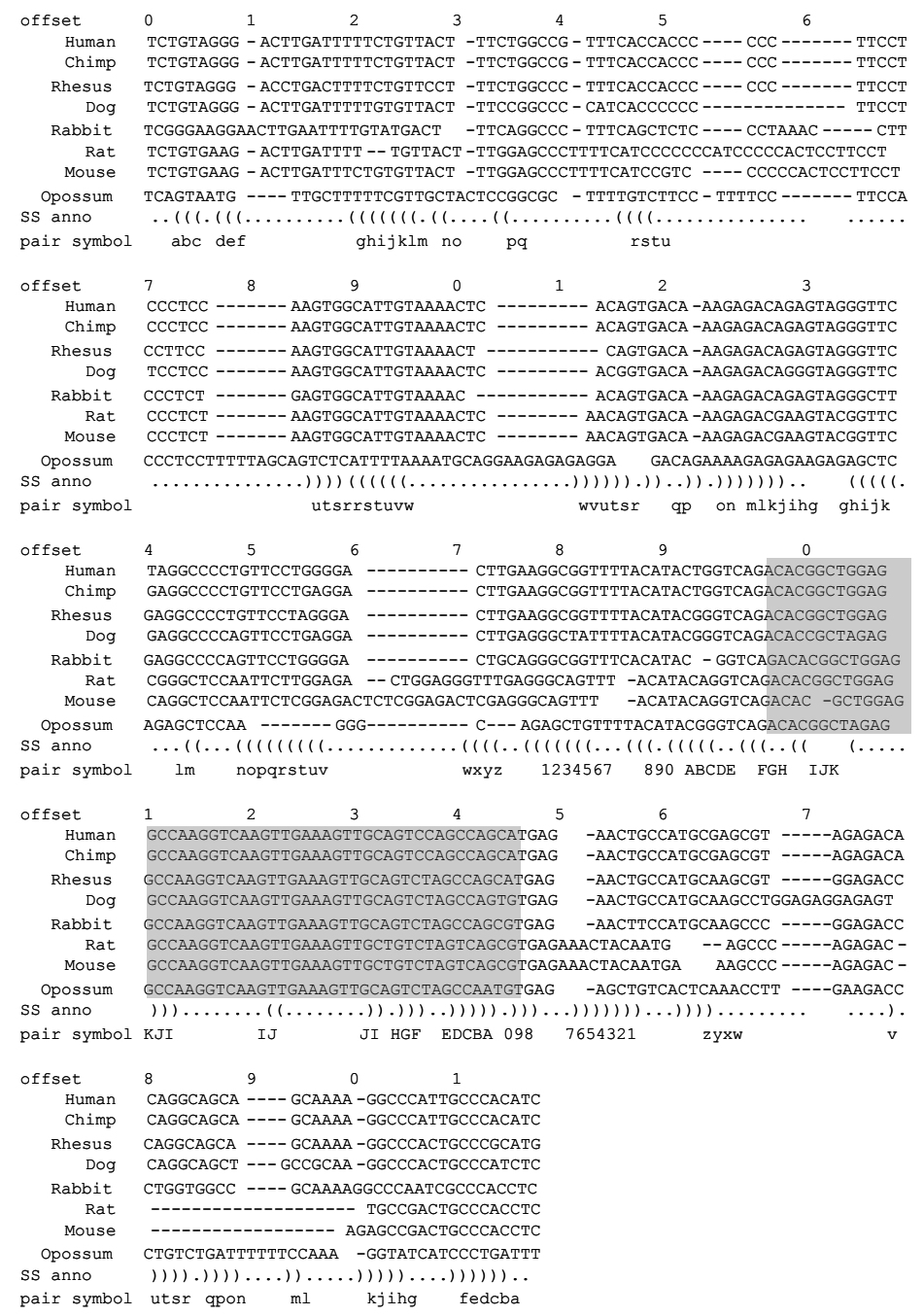

B

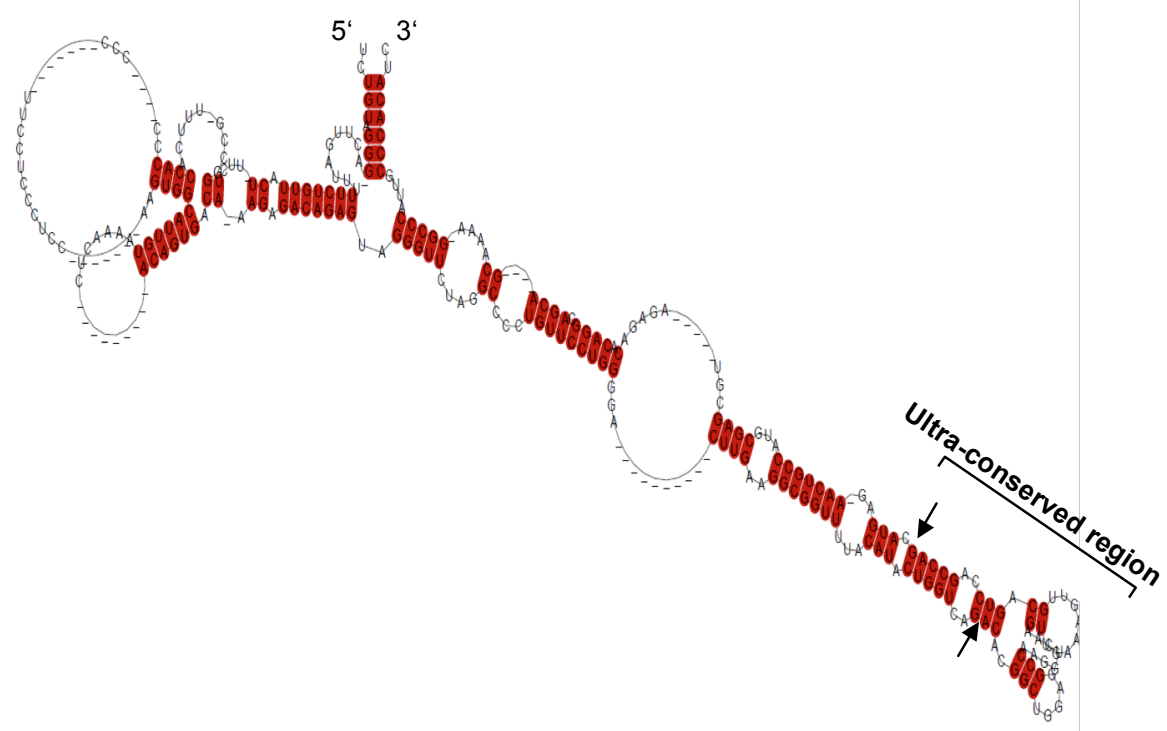

C

human: HIT18844

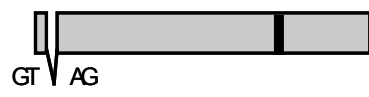

mouse: AK033508

mouse: BC046496

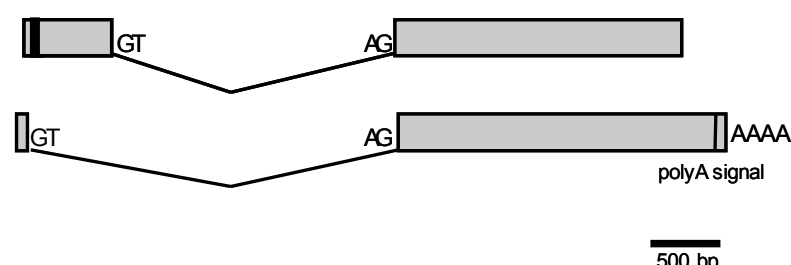

Sasaki et al. Fig. 3 